\title{
Interdisciplinary rehabilitation in morbidly obese subjects: an observational pilot study
}

\author{
E. Clini, F. Prato, M. Nobile, M. Bondi, B. Serri, C. Cilione, D. Lugli
}

ABSTRACT: Interdisciplinary rehabilitation in morbidly obese subjects: an observational pilot study. E. Clini, F. Prato, M. Nobile, M. Bondi, B. Serri, C. Cilione, D. Lugli. Background and aim. To assess the clinical effectiveness of a interdisciplinary rehabillitation programme (CR), in a population of morbidly obese subjects we have undertaken a observational study.

Methods. The study included fifty-nine adult subjects (18 M, 60 \pm 10 years, BMI 47 \pm 8 ) with sleep-disturbance related symptoms and disabilities. Assessment and correction of sleep disordered breathing (SDB) abnormalities, improvement of exercise tolerance, body weight and associated psychological features were the aims of this CR, which has been carried out over a 1 month period.Lung functions, apnea/hypopnea index (AHI), 6-minute walking distance (6MWD), body weight (BW), quality of life by means of Sat-P questionnaire and serum metabolic data has been recorded at baseline (T0), at the end (T1) and 6 months after (T2) the CR.

Results. The percentage of patients with AHI $>10$ declined from $65 \%$ (at T0) to $20 \%$ (at both T1 and T2). 6MWD and BW significantly improved $(p<0.005)$ at T1 and still maintained at $\mathrm{T} 2$; a significant relationship $(r=0.379, p<0.01)$ has been found between changes of $B W$ and 6MWD recorded in between T0 and T2. Sat-P item scores dealing with sleep efficiency, problem solving, and social interactions improved $(\mathbf{p}<0.01)$ at $\mathrm{T} 1$ and still maintained at $\mathbf{T} 2$.

Conclusions. This hospital-based CR provides indication for effectiveness in advanced morbidly obese subjects and warrants further controlled trials to confirm the results. Monaldi Arch Chest Dis 2006; 65: 2, 89-95.

Keywords: Obesity, exercise, quality of life, nutrition, apnea/hypopnea.

University of Modena-Reggio Emilia and Dpt. of Pulmonary Rehabilitation, Villa Pineta Hospital, Pavullo (MO). Italy.

Correspondence: Prof. Enrico M.Clini; University of Modena and Villa Pineta Hospital; Dpt. of Pulmonary Rehabilitation; Via Gaiato 127; 41020 Pavullo (MO), Italy; e-mail: clini.enrico@unimore.it

\section{Introduction}

Obesity has become a major health problem over recent decades; over-nutrition is second only to smoking as a general cause of death $[1,2]$. The prevalence of this condition has rapidly increased in USA [3] and in western Europe [4].

Obesity induced health risk results from several comorbid conditions $[5,6]$ such as metabolic disorders, cardiovascular diseases, neoplasm and respiratory syndromes [7], obstructive sleep apnea (OSA) being one of the most important and frequently linked disorders [8].

Apart from the surgical approaches in advanced obesity [9], medical treatments have been proposed in mild to moderate degrees of obesity with the goal of reducing excess weight. These treatments, mainly consist of behavioural therapies (eating habits, low calories diets, and physical activity) with the addition of nasal CPAP (nCPAP) among those patients presenting obstructive sleep apnea (OSA), which has been associated with variably consistent weight loss and improvements to the apnea/hypopnea index (AHI) and/or the pulmonary mechanics [10]. However, the major aim of this conservative therapy is not only to focus at- tention on $5-10 \%$ loss of body weight, but also on the long-term maintenance of body weight reduction as well as of any other favourably changed behaviour [11].

To our knowledge, there is no clear information on the possibility that an interdisciplinary conservative approach may favour improvements, and possibly long-term maintenance, in severely obese subjects. Therefore, we have undertaken an observational pilot study in a rehabilitation environment on a population of severely obese individuals, suffering from sleep disturbances and advanced disability, and consecutively admitted to an inpatient programme (CR).

The aim of this study was to assess several clinical outcomes in these patients at the end of a 1-month CR and 6 months after discharge as the latest follow-up.

\section{Methods}

The study procedures were conducted in accordance with the Helsinki declaration. All the subjects gave their informed consent to participate in this programme. 


\section{Patients}

The in-hospital programme was designed for morbidly obese adult subjects (range 26 to 76 years) with BMI $\geq 35 \mathrm{~kg} / \mathrm{m}^{2}$ who have previously failed to lose weight in a outpatient setting and who suffer from several medical complications (hypertension, secondary diabetes, etc.) because of their obesity. In particular, all the admitted patients referred to suffer from sleep disturbances and initially scored $>6$ at the Epworth Sleepiness Scale (ESS) [12]. $71 \%$ of the patients presented with binge eating disorders as defined by the DSM-IV diagnostic criteria [13] and confirmed by the Eating Disorder Inventory (EDI-2) test [14] adapted for Italian people [15]. In addition, $61 \%$ of the patients were affected by moderate/major depression according to the Beck's scale [16]: so far, they assumed daily antidepressant agents. Patients with bulimia nervosa and obesity secondary to endocrine diseases were excluded. Table 1 shows demographic, anthropometric and social characteristics of patients at study entry.

\section{Interdisciplinary rehabilitation program}

The structured and interdisciplinary CR programme was initiated in our department in January 2003. The hospital medical and ethical committees approved the purpose and the content of the programme which was created and properly modified in the year before. Interdisciplinary staff delivered the in-hospital programme over a 1-month period and it involved individual as well as group therapy sessions. The staff is composed of a pulmonologist, endocrinologist, dietician, physical therapist and psychologist, while other allied health professionals (namely nurse cohordinator and social assistant) implemented daily activities; the chief of the department is actually the coordinator and the responsible of the $\mathrm{CR}$.

Table 1. - Demographic, anthropometric and social characteristics of patients at study entry. Data is expressed as numbers (\%) or means (SD)

\begin{tabular}{lc}
\hline & Cases (n=59) \\
\hline Sex (M / F) & $18(31) / 41(69)$ \\
Age (years) & $60(10)$ \\
Weight (kg) & $122(28)$ \\
BMI (kg x m $\left.{ }^{2}\right)$ & $47(8)$ \\
Binge Eating Scale $\geq 17$ (Yes / No) & $52(88 \%) / 7(12 \%)$ \\
\hline Familiar Status & \\
Married & $34(58)$ \\
Unmarried & $25(32)$ \\
\hline Job Condition & \\
Employed & $5(8)$ \\
Unemployed & $7(12)$ \\
Housewife & $7(12)$ \\
Retired & $40(68)$ \\
\hline
\end{tabular}

Abbreviation: BMI: Body Mass Index.
The short-term aims of the programme were either assessment and correction of the sleep disordered breathing (SDB) abnormalities, reduction of body weight, improvement of both exercise tolerance and of the associated psychological features. The aim in the long-term follow-up was to maintain any of the previously described goals.

A polisomnographic assessment of apnea/hypopnea index (AHI) was performed, according to the current national guidelines [17], at baseline and then repeated at 6-months. Appliance, setting and prescription of nCPAP has been considered in obese patients with AHI >20; they were all encouraged and trained to continue this treatment at home. Overnight pressure levels has been set by means of the autoadjusting titration system as described elsewhere [18]; patient's adherence and compliance have been checked during a six-night consecutive recording.

A low-calorie diet was specifically tailored on the basis of individual patient's needing and according to his/her metabolic functions explored. Overall, this diet was balanced with $30 \%$ fats, $55 \%$ carbohydrates and $15 \%$ proteins divided into two meals and two snacks. Nutritional education was provided twice per week by a dietician.

Exercise consisted of twice daily one-hour sessions of moderate to low intensity activities including: i) supervised incremental exercise on a cycloergometer; training modality consisted of both progressively increasing load (up to 70-80\% of the maximal load reached on the incremental test carried out at admission; a dyspnea score score below 5 determined the ability of the single patient to increase load) and time (up to 30 consecutive minutes starting from a minimum of 10 ); ii) abdominal, upper and lower limb muscle strength activities, lifting weights (up to $3 \mathrm{~kg}$ ) and circling shoulders and full arms. Instructions for energy conservation, stress management and symptoms control were also delivered to patients. Patients were encouraged to perform their usual daily life activities on their return home. In addition, written advice on how to maintain physical fitness was given to patients upon discharge; however, strategies to perform supervised exercise programmes at home were not implemented during the follow-up.

Twice weekly sessions of psychotherapy were carried out during their hospital stay. The behavioural therapy consisted of sessions dealing with issues of self-control, cognitive restructuring reinforcement and relapse prevention. No other structured interventions was delivered to patients during this period.

\section{Study design}

The study was a prospective observational trial. After completing the in-hospital CR, patients were discharged to return home. A brief non-structured telephone interview was performed in between (about 3 months) discharge and the latest follow-up; at this time patients were subsequently hospitalised to take measurements.

Assessments were therefore performed baseline within the first three days from admission 
(T0), at hospital discharge (T1), and 6 months later (T2).

\section{Measurements}

\section{RESPIRATORY FUNCTION}

Lung volumes $\left(\mathrm{FEV}_{1}, \mathrm{FVC}, \mathrm{VC}\right)$ were recorded by means of a spirometer (Masterscope Jaeger GmbH, Hochberg-Germany). The predicted values according to Quanjer [19] were used. Arterial blood to obtain $\mathrm{PaO}_{2}, \mathrm{PaCO}_{2}$ and $\mathrm{pH}$ by an automated analyser (Mod.850, Chiron Diagnostics Co., Medfield-USA) was sampled at the radial artery while patients, in the sitting position, were breathing room air for at least one hour.

The apnea/hypopnea index (AHI) was calculated from the full polisomnography tracing [20].

\section{WEIGHT AND EXERCISE PERFORMANCE}

Body weight (BW) was assessed in the morning after waking-up by means of a electronically calibrated platform scale with remote display and high load capacity (SECA mod.635, HamburgGermany).

The timed walking distance in metres was assessed by the 6 minute test (6MWD) as recommended [21]. Before and at the end of effort, patients were asked their perceived dyspnea and leg fatigue by pointing a number or phrase on a 10point modified Borg scale [22].

\section{QUALITY OF LIFE AND PSYCHOLOGICAL FUNCTION}

A generic measure of the patients' quality of life was recorded by means of the Sat-P questionnaire [23]. Psychological functions were assessed at T0 and T2; the Italian versions of the Minnesota Multiphasic Personality Inventory 2 (MMPI-2) [24] and the Eating Disorder Inventory 2 (EDI-2) [15] were tested to assess the patients' personality and eating disturbances, respectively. The patients' binge eating severity was then scored as previously described and published [25].

\section{MetABOLIC DATA}

Plasma total cholesterol, triglycerides and uric acid were measured according to the most common laboratory methods; glicate-Haemoglobin (glicate- $\mathrm{Hb}$ ) was also measured in those patients with associated secondary diabetes $(n=22)$.

\section{Analysis}

Unless otherwise indicated, all data is presented as mean \pm 1 standard deviation (SD). Analysis was performed using a specific software package (SPSS 8.0 for Windows).

ANOVA for repeated measures was used to test the differences between times. Contrasts among times were evaluated by means of $t$-test with Bonferroni adjustment. Sperman's correlation coefficient was then calculated among all the variables' changes obtained at different times. A $p$ value less than 0.05 was considered to be significant.

\section{Results}

All the included patients completed their follow-up. Table 1 shows demographic, anthropometric and social characteristics of the studied population: 49 obese patients $(83 \%)$ had a BMI $>40$ $\mathrm{kg} / \mathrm{m}^{2}$. Upon admission, the threshold limit of 17 at the binge eating score measure [24] was recorded in most $(88 \%)$ of the subjects. None of the admitted patients underwent surgical treatment for obesity; all of them experienced previous dietary interventions without any other structured comprehensive in-hospital approach.

\section{RESPIRATORY FUNCTION}

Analysis of spirometry revealed that $20 \%$ of patients showed chronic airway obstruction due to asthma and/or COPD. Changes over time in lung volumes, gas exchange and $\mathrm{AHI}$ are shown in table 2. As the most relevant observation, $\mathrm{PaO}_{2}$ in resting condition significantly rose over time, whereas AHI decreased after the 1-month pro-

Table 2. - Time course of respiratory functions in the population studied. Data is expressed as means (SD)

\begin{tabular}{|c|c|c|c|}
\hline & \multicolumn{3}{|c|}{ Cases $(n=59)$} \\
\hline & TO & $T 1$ & $T 2$ \\
\hline $\mathrm{FEV}_{1}(\%$ pred $)$ & $86(27)$ & na & $87(42)$ \\
\hline FVC (\% pred $)$ & $90(22)$ & na & $91(21)$ \\
\hline $\mathrm{VC}(\%$ pred $)$ & 92 (14) & na & 93 (15) \\
\hline $\mathrm{PaCO}_{2}(\mathrm{mmHg})$ & 42 (6) & $39(8) \S$ & 41 (3) \\
\hline $\mathrm{PaO}_{2}(\mathrm{mmHg})$ & 64 (9) & $68(13)$ & 68 (7) $\S$ \\
\hline $\mathrm{AHI}$ & $40(39)$ & 28 (9) \# & 25 (4) \# \\
\hline
\end{tabular}

na - not available

$\S p<0.05$ versus Baseline

\# $\mathrm{p}<0.01$ versus Baseline

Abbreviations: $\mathrm{FEV}_{1}$ : Forced Expiratory Volume in one second; FVC: Forced Vital Capacity; VC: Vital Capacity; $\mathrm{PaO}_{2}$ : Arterial Oxygen Tension; $\mathrm{PaCO}_{2}$ : Arterial Carbon dioxide Tension; AHI: Apnea Hypopnea Index. 
gramme (T1) and maintained at 6 months (T2). As a whole, at baseline, $65 \%$ of obese patients presented a AHI >10, whereas this percentage decreased to $20 \%$ both at $\mathrm{T} 1$ and $\mathrm{T} 2.14$ obese patients with $\mathrm{AHI} \geq 20$ at $\mathrm{T} 0$ were prescribed and trained to domiciliary nCPAP; all of them improved both $\mathrm{PaO}_{2}$ and $\mathrm{AHI}$ during the follow-up.

\section{WEIGHT AND EXERCISE PERFORMANCE}

BW and exercise capacity as assessed by 6MWD significantly improved over time (see figure 1); in particular, improvement of both outcomes at $\mathrm{T} 1$ still maintained at T2. Short-term improvement of exercise tolerance has been further confirmed by reduction of symptoms score (from $4.3 \pm 2.0$ to $2.1 \pm 1.8$ for dyspnea and from $4.5 \pm 2.6$ to $2.4 \pm 1.5$ for leg fatigue, $\mathrm{p}<0.005$ ) as assessed by Borg scale at peak effort. At T2, 24 obese patients $(41 \%)$ significantly reduced (by more than 5\%) their BW further (ranging in between 5.1 to $20 \%$ of the value reached at T1); among the remaining 35 patients, $18(51 \%)$ regained, 1 (3\%) maintained (less than 1\% change), and 16 $(45 \%)$ reduced BW (range in between 1 to $5 \%$ ) their $\mathrm{BW}$ at $\mathrm{T} 0$ (for details see also figure 2). A significant positive correlation $(\mathrm{r}=0.379, \mathrm{p}<0.01)$ has been found between changes of BW and 6MWD recorded in between $\mathrm{T} 0$ and $\mathrm{T} 2$.

\section{QUALITY OF LIFE AND PSYCHOLOGICAL FUNCTION}

The evaluation of patients' generic quality of life by means of the Sat-P questionnaire has shown that almost all the items (those dealing with sleep, dietary behaviour, resistance to fatigue, mobility, activity, mood, emotions, participation and selfcontrol) significantly improved $(\mathrm{p}<0.05)$ at $\mathrm{T} 1$. Overall, they turned down to baseline at T2 (detailed data not showed); however, the item scores dealing with sleep efficiency (from $57 \pm 29$ to $75 \pm 20$ and to $63 \pm 26$, at $\mathrm{T} 0$, T1 and $\mathrm{T} 2$ respectively) problem solving (from $65 \pm 31$ to $84 \pm 17$ and to $74 \pm 23$ ), and social interactions (from $68 \pm 35$ to $87 \pm 17$ and to $79 \pm 19)$ improved at $\mathrm{T} 1$ and still maintained at $\mathrm{T} 2$.

Finally, 6-month comparison of psychological function tests' scores has shown that EDI-2 components did not change, whereas angry and familiar components of MMPI-2 significantly improved $(p<0.05)$ over time (detailed data not showed). Moreover, Binge Eating Score (BES) $>17$ (suggesting the presence of a significant eating disturbance) [25] was recorded in $52(88 \%)$ and $30(51 \%)$ of patients at T0 and $\mathrm{T} 2$, respectively.

\section{Metabolic DATA}

Table 3 shows metabolic data measured across the study period.
A significant decrease has been recorded for both Glicate-Hb and Uric Acid at T1, whilst these improvements also maintained at $\mathrm{T} 2$.

\section{Discussion}

This hospital-based interdisciplinary rehabilitation programme provides useful benefits in mor-

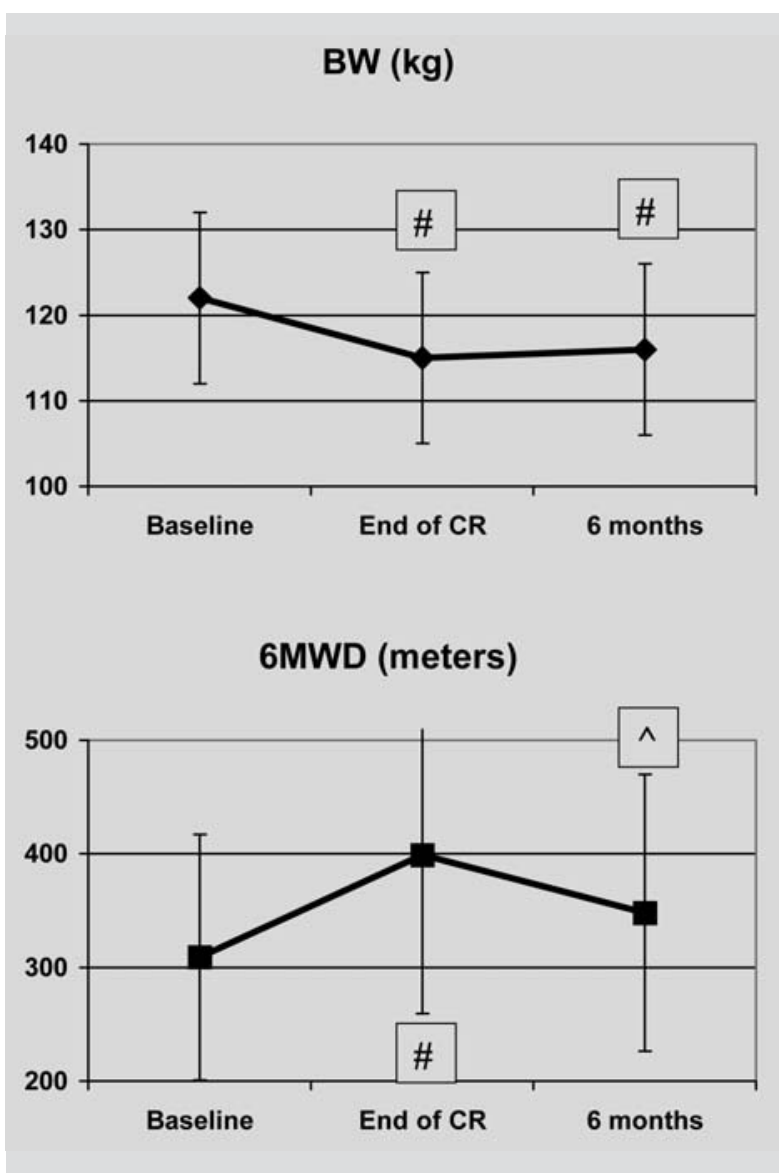

Fig. 1. - Change over time of body weight (upper panel) and exercise performance (lower panel) in the population studied (\# $\mathrm{p}<0.005$ versus $\mathrm{T} 0 ;^{\wedge} \mathrm{p}<0.01$ versus $\left.\mathrm{T} 0\right)$.

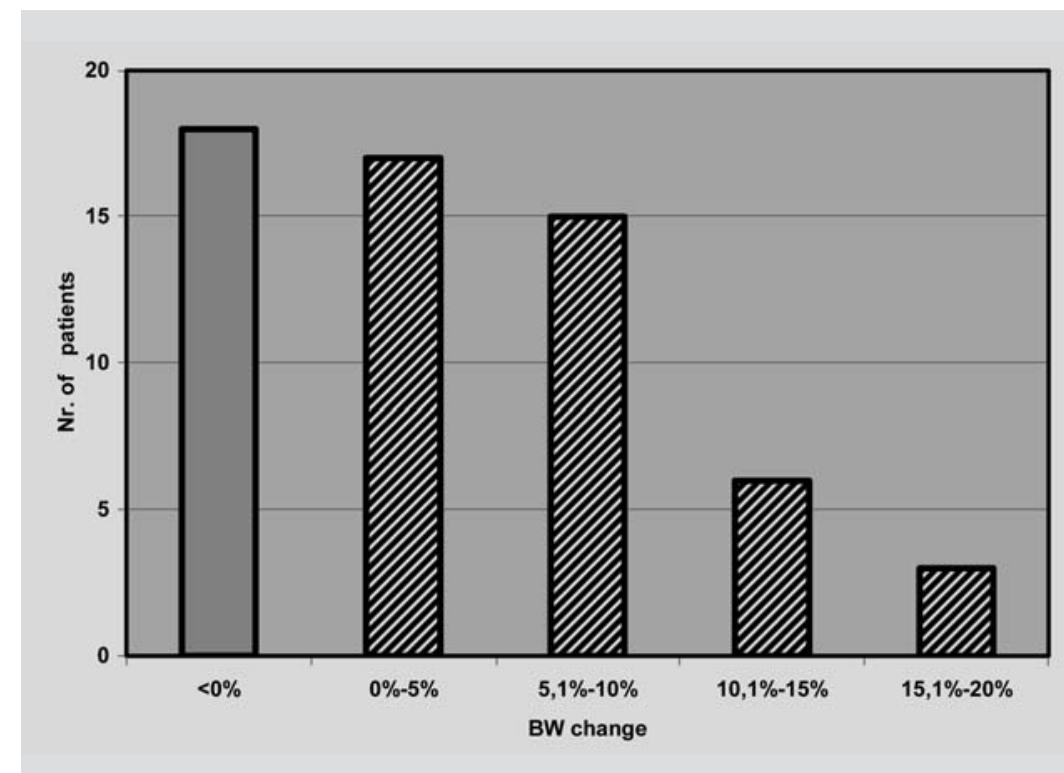

Fig. 2. - Patients distribution (number) according to the percentage reduction of BW during the follow-up (in between $\mathrm{T} 1$ and T2). Solid bar represents patients who regained their BW over time. 
Table 3. - Time course of metabolic data in the population studied. Data is expressed as means (SD)

\begin{tabular}{|c|c|c|c|}
\hline & \multicolumn{3}{|c|}{ Cases $(n=59)$} \\
\hline & TO & $T 1$ & $T 2$ \\
\hline Glicate Haemoglobin $(\mathrm{g} / \mathrm{L}) *$ & 6.4 (1.4) & $5.9(0.8) \S$ & $6.0 \quad(0.9)$ \\
\hline Total cholesterol (mg/dL) & $225.7(54.3)$ & $179.3(33.2) \#$ & $204.4(41.4)$ \\
\hline Triglicerides (mg/dL) & $144.5(53.0)$ & $140.2(47.7)$ & $141.3(78.2)$ \\
\hline Uric Acid (mg/dL) & $5.3(1.3)$ & $5.5(1.2)$ & $4.8(1.1)$ \\
\hline
\end{tabular}

bidly obese subjects. Corrrections of sleep disturbances, improvement of body weight, exercise capacity and psychological functions have been obtained in a large amount of patients on a short-tem basis (after 1-month of treatment) and these benefits also maintained thereafter (at the latest 6month follow-up). In particular, over time loss of body weight and gain in exercise performance significantly correlated each other in these advanced morbidly obese subjects showing disability.

Little data exists in literature describing the effect of interdisciplinary and structured programs delivered in-hospital to the most advanced and disabled adult obese subjects failing several previous conservative attempts to improve their condition. To our knowledge, only one very recently published paper described the short and long term effectiveness of a innovative interdisciplinary cognitive-nutritional approach to treat obesity [26].

In that observational study [26] authors have studied the long-term (up to 5 years) effects of their 6-weeks hospital programme, combining daily nutritional therapy with physical exercise and relaxation sessions, delivered to morbidly obese patients with a BMI of $40 \pm 1 \mathrm{~kg} / \mathrm{m}^{2}$. They found that, five years later, $20 \%$ of patients maintained their initial weight loss, whereas $25 \%$ of them further decreased their body weight. Overall, the weight change significantly correlated with the degree of psycho-social difficulties; moreover, serum metabolic data improved over time in those who had lost more weight.

Although referred to a very similar study population, any comparison between data obtained in that study [26] and in our study seems to be difficult for several reasons. First, the follow-up is different in length and management; indeed, Golay et al. [26] hospitalised patients for one day every 4 months for motivational purposes and, in addition, obese subjects were followed up 4 times a year by their private GP. Second, and most importantly, it is clear that weight loss has been considered as the only major end point of their programme: the specified aims for the short and long-term effects in our study were more than the weight change alone. Taken as a whole, our CR programme aimed to assess and correct OSA, reduce body weight, improve exercise tolerance and positively influence any of the psychological features commonly associated to these patients.
Assessment and correction of sleep disordered breathing (SDB) appear to be two important goals needed to achieve in the most advanced morbidly obese; as a matter of fact, a $10 \%$ increase in body weight has been shown to predict a 6 -fold increase in the odds of developing moderate to severe disturbances in a longitudinal population-based study [27], and also it is known that $70 \%$ of patients with OSA are obese [7], while hypoventilation commonly complicating adult obesity is associated with excess morbidity and mortality [28]. Despite the fact that in our study all the admitted patient scored above 6 at the ESS, nonetheless our approach to look for any significant SDB allowed us to confirm that more than $60 \%$ of patients presented these disturbancies (AHI >10), 14 of them (37\%) needing a nCPAP overnight correction. This in-hospital management of obese patients certainly resulted in a significant short-term improvement and long-term maintenance of SDB and arterial oxygenation in the affected patients (see Results section and table 2 ).

The overall change and maintenance in BW and exercise tolerance are two additional important goals achieved by our programme (see figure 1). In particular, at difference with our study, the previously published study in similar patients [26] did not underline the precise role of exercise enhancement in these obese, nor it presented any accurate description on how the patients were trained. To confirm these findings we have also found a significant correlation between long-term changes of $\mathrm{BW}$ and exercise, thus suggesting a really spontaneously improved and/or modified lifestyle in these adult obese. About half of our population regained $\mathrm{BW}$ at the latest follow-up (see figure 2), which accounts for a proportion similar to that found over 5 years in the study by Golay et al. [26].

These latter effects are likely to consistently reflect the significant improvement in the generic quality of life and in the psychological functions explored. Overall, the items of the Sat-P questionnaire scores improved at the end of CR but turned baseline thereafter; however, those dealing with sleep quality, problem solving and participation were still being maintained at follow-up, thus suggesting a modified lifestyle according to the benefits obtained in terms of SDB correction, BW loss and exercise enhancement. 
Furthermore, long-term reassessment of personality test (MMPI-2) has shown in addition a significant improvement in the angry and familiar component scores, whereas the threshold limit of the Binge Eating Score (BES) measure [25] has been recorded in significantly less patients after 6 months. This further result it is likely to benefit these subjects. Indeed, as previously suggested, these factors are often interconnected: it is well known that patients with eating disorders do not respect the frequency or timetable of meals, and are more prevalent in populations with psycho-social difficulties [29-31]. In the study by Golay et al. [26] those patients diagnosed as having eating behaviour problems (66\%) frequently reported loneliness, family and couple problems, and mourning as reasons for over-eating; moreover, a significantly higher number identified emotional liability, depression $(52 \%)$ or anxiety $(58 \%)$ rather than hunger as the trigger.

Among the metabolic data taken in our studied sample, total serum cholesterol and glicate- $\mathrm{Hb}$ have shown to be reduced at the end of CR, while trended to recover (but not reached) the baseline value at the end of follow-up. This overall effect is certainly due to the more strict dietary intake during hospitalisation; however, the proportion of patients who did not regain weight at the follow-up also maintained their cholesterol and glicate- $\mathrm{Hb}$ stable, thus confirming previous finding in successfully treated obese [26]. Additionally, in our experience, the number of patients with type II diabetes needing anti-diabetic drugs reduced over time (from 22 at T0 to 8 at T2) according to the reduction of patients having a glicate-Hb above the limit of $5.7 \mathrm{mg} / \mathrm{dL}$. Various authors have described the efficacy of weight loss on blood sugar and insulin levels, and insulin resistance [32, 33]. Maintaining the metabolic benefit of weight loss confirm an overwhelming long-term metabolic improvement in comparison with the natural evolution of obesity towards type 2 diabetes mellitus $[34,35]$.

The reduction of the amount of drugs used per patients cannot be considered to be a trivial effect. In our obese subjects, the use of antidepressants reduced as well in the follow-up, thus confirming once more the overall positive effect on the patient's lifestyle that our CR could have determined.

The present study has some important limitations which need to be carefully evaluated by the reader. First, the protocol is not performed on a randomised parallel group basis. However, when admitting advanced obese patients who have already failed previous attempts to benefit from conservative therapies, it seems rather hard to differentiate the programme or to deny them any of the included treatments. Second, the relatively small sample size, although it is similar to that of the previous comparable study [26], and the relatively short follow-up period could limit the value of the obtained results. Nonetheless, all the subjects included completed the CR and were successfully followed over time thus making the obtained results possibly reproducible on a larger population of similar individuals and for longer time. Third, this interdisciplinary approach fails to identify which of the various interventions proved most efficacious in achieving the stated objectives; nonetheless, all of the various management initiatives might be critical (and not necessarily redundant) in reaching the most important outcome for these patients, which is the long-term weight loss. Finally, the long in-hospital stay is a potentially high source of increasing costs of the rehabilitation programme. However, our programme does not appear to be any more expensive than others previously published [26]. Although a specific analysis might have arisen advantages and/or disadvantages in terms of costs, this was not the aim of the present study.

In conclusion, this hospital-based interdisciplinary rehabilitation programme has proven to provide indications for effectiveness in a group of morbidly obese subjects. Despite the fact that there are no international [36] or local [37] documents included in the detailed guidelines for a comprehensive rehabilitation in these subjects, it is likely that this treatment may really favour obese patients who had already failed several attempt to improve their status. Our findings warrant future controlled trials with a longer follow-up to confirm the results.

\section{References}

1. McGinnis JM, Foege WH. Actual causes of death in the United States. JAMA 1993; 270: 2207-2212.

2. Manson JE, Willett WC, Stampfer MJ, et al. Body weight and mortality among women. NEJM 1995; 333: 677-685.

3. Stunkard AJ. Current views on obesity. Am J Med 1992; 100: 230-236.

4. World Health Organization. Obesity: preventing and managing the global epidemic. Report of a WHO consultation on obesity. Geneva, 3-5 June 1997: 27-29.

5. Pi-Sunyer FX. Medical hazards of obesity. Ann Intern Med 1993; 119: 655-660.

6. World Health Organization. The world health repport 2002: reducing risk, promoting healthy life. Geneva, 2002.

7. Bates DV. Respiratory Function in Disease, 3rd Edn. Philadelphia: WB Saunders 1989; 100-102.

8. Guilleminault C. Clinical features and evaluation of obstructive sleep apnoea. In: Kryger MH, Roth T, Dement WC (eds). Principles and Practice of Sleep Medicine. Philadelphia: WB Saunders 1994.

9. Kolanowsky J. Surgical treatment for morbid obesity. Br Med Bull 1997; 53: 433-444.

10. Barvaux BA, Aubert G, Rodenstein DO. Weight loss as a treatment for obstructive sleep apnoea. Sleep Med Rev 2000; 4: 435-452.

11. Goldstein DJ. Beneficial health effects of modest weight loss. Int J Obes 1992; 16: 397-415.

12. Johns MW. A new method for measuring daytime sleepiness: the Epworth sleepiness scale. Sleep 1991; 14: 540-545.

13. American Psychiatric Association. Diagnostic and statistical manual of mental disorders (DSM-IV). American Psychiatric Press, Washington DC. 1994.

14. Garner DM. Eating Disorder Inventory, $2^{\text {nd }}$ professional manual. Psychological Assessment Resources, New York. 1990. 
15. Rizzardi M, Trombini G, Trombini-Corazza E. Eating Disorder Inventory 2 test (italian version). O.S. ed, Firenze. 1995.

16. Beck AT, Ward CH, Mendellson M, Mock J, Erbaugh J. An inventory for measuring depression. Arch Gen Psychiatr 1961; 4: 5611-571.

17. AIPO study group on Sleep-related Disturbancies. Italian guidelines for polysomnographic reports and cardio-resppiratory monitoring in adults with suspected sleep apnea syndrome (SAS). Rassegna Patologia Apparato Respiratorio 2003; 18: 305-308.

18. Masa JF, Jimenez A, Duran J, et al. Alternative methods of titrating Continuous Positive Airway Pressure. Am J Respir Crit Care Med 2004; 170: 1218-1224.

19. Quanjer PH. Working Party on "Standardization of lung function test". Bull Eur Physiopathol Respir 1983; 19 (Suppl5): 7-10.

20. Report of an American Academy of Sleep Medicine Task Force. Sleep-related breathing disorders in adults: recommendations for syndrome definition and measurement techniques in clinical research. Sleep 1999; 22: 667-89.

21. American Thoracic Society. Statement: Guidelines for the six-minute walk test. Am J Respir Crit Care Med 2002; 166: 111-117.

22. Borg GAV. Psychophysical basis of perceived exertion. Med Sci Sports Exerc 1992; 14: 377-381.

23. Majani G, Pierobon A, Giardini A, Callegari S. Satisfaction Profile (SAT-P) in 732 patients: focus on subjectivity in HRQL assessment. Psychol Health 2000; 15: 409-422.

24. Sirigatti S, Pancheri P. Minnesota Multiphasic Personality Inventory 2 test (italian version). O.S. ed, Firenze. 1995.

25. Timmerman GM. Binge Eating Scale: further assessment of validity and reliability. J Appl Biobehav Res 1999; 4: 1-12

26. Golay A, Buclin S, Ybarra J, et al. New interdisciplinary cogniitive-behavioural-nutritional approach to obesity treatment. Eat Weght Disord 2004; 9: 29-34.

27. Peppard PE, Young T, Palta M, Dempsey J, Skatrud J. Longitudinal study of moderate weight change and sleep-disorderd breathing. JAMA 2000; 284: 30153021.

28. Nowbar S, Burkart KM, Gonzales R, et al. Obesity-associated hypoventilation in hospitalized patients: prevalence, effects and outcome. Am J Med 2004; 116: 1-7.

29. Walsh BT, Devlin MJ. Eating disorders: progress and problems. Science 1998; 280: 1387-1390.

30. Mussell MP, Mitchel JE, De Zwaan M, Crosby RD, Seim HC, Crow SJ. Clinical characteristics associated with binge eating in obese females: a descriptive study. Int J Obes Relat Metab Disord 1996; 20: 324-331.

31. Raymond NC, Mussell MP, Mitchell JE, De Zwaan M, Crosby RD. An age-matched comparison of subjects with binge eating disorder and bulimia nervosa. Int $J$ Eat Disord 1995, 18 (Suppl. 29): 135-143.

32. Purnell JQ, Kahn SE, Albers JJ, Nevin DN, Brunzell JD, Schwartz RS. Effect of weight loss with reduction of intra-abdominal fat on lipid metabolism in older men. J Clin Endocrinol Metab 2000; 85 (Suppl. 3): 977-982.

33. Olefsky J, Reaven GM, Farquhar JW. Effects of weight reduction in obesity: studies of lipid and carbohydrate metabolism in normal and hyperlipoproteinemic subjects. J Clin Invest 1974; 53: 64-76.

34. Sorbis R, Petersson BG, Nilsson-Ehle P. Effects of weight reduction on plasma lipoproteins and adipose tissue metabolism in obese subjects. Eur J Clin Invest 1981; 11: 491-498.

35. Petersen KF, Hendler R, Price T, et al. 13C/31P NMR Studies on the mechanism of insulin resistance in obesity. Diabetes 1998; 47: 381-386.

36. National Institutes of Health National Heart, Lung, and Blood Institute in cooperation with the National Institute of Diabetes and Digestive and Kidney Diseases. Clinical Guidelines on the Identification, Evaluation, and Treatment of Overweight and Obesity in adults. The Evidence Report. NIH Publication No. 98-4083 September 1998.

37. Italian Ministry of Health. Recommendations for the prevention, diagnosis and treatment of overweight and related diseases. 2004 (http://www.sio-lombardia.org/ $\mathrm{php} / \mathrm{html} / \mathrm{aaaa} / \mathrm{ministero/Consensus.pdf).}$

\section{APPENDIX}

\section{Epworth Sleepiness Scale [ESS]}

A simple 6-items scale each scoring 0 to 3, with a possible range of $0-18$. Items describe the patient's sleepiness in different conditions of daily living. Total score correlates with the severity of sleepiness, being normal below 6 .

\section{Eating Disorder Inventory test [EDI-2]}

A self-administered interview consisting of 91 "true or false" items [graded from "always" to "never"] grouped into 11 scales. It describes the individual's symptoms related to anorexia and bulimia.

\section{Beck Depression Scale [BDS]}

A self-administered scale consisting of 21 items each describing 4 level of gradually increasing severity to assess specific manifestations related to the individual's depression (excluding anxiety).

\section{Satisfaction Profile questionnaire [Sat-P]}

A self-administered generic questionnaire which provides a satisfaction profile over 32 daily life aspects with reference to the last month. It provides 3210 -cm horizontal VAS and 5 factor scores all ranging from 0 (lowest satisfaction) to 100 (highest satisfaction)

\section{Minnesota Multiphasic Personality Inventory [MMPI-2]}

A self-administered interview consisting of 567 "true or false" items grouped into several scales. It describes individual's personality and emotional disorders. Score of 65 is considered the threshold limit between normality and pathology.

\section{Binge Eating Scale [BES]}

A self-administered 16-items ( 8 describing behavioral manifestation and 8 describing associated feelings and cognitions) interview to measure binge eating severity. Each item consists of 4 statements that reflect a range of severity (o to 3 ), with a possible range $0-46$. 